\title{
REDUCED ORDER MODELLING OF LINEAR MIMO SYSTEMS USING GENETIC ALGORITHM
}

\author{
Parmar, G.; Mukherjee, S. \& Prasad, R. \\ Department of Electrical Engineering, Indian Institute of Technology, Roorkee-247667, \\ Uttarakhand, India \\ E-Mail: gp555dee@iitr.ernet.in; girish_parmar2002@yahoo.com
}

\begin{abstract}
In this paper, an algorithm for order reduction of linear multivariable systems is proposed using the combined advantages of the dominant pole retention method and the error minimization by Genetic algorithm. The denominator of the reduced order transfer function matrix is obtained by retaining the dominant poles of the original system while the numerator terms of the lower order transfer matrix are determined by minimizing the integral square error in between the transient responses of original and reduced order models using Genetic algorithm. Each element of the transfer function matrix of the original system is considered separately. The reduction procedure is simple and computer oriented. The proposed algorithm guarantees stability of the reduced order transfer function matrix if the original high order system is stable and is having superior features, including easy implementation and good computational efficiency. The proposed algorithm has been applied successfully to the transfer function matrix of a $10^{\text {th }}$ order two-input two-output linear time invariant model of a practical power system. The performance of the algorithm is tested by comparing the relevant computer simulation results.

(Received in November 2006, accepted in May 2007. This paper was with the authors 1 month for 1 revision.)
\end{abstract}

Key Words: Dominant Pole, Genetic Algorithm, Order Reduction, Power System

\section{Nomenclature}

$\begin{array}{ll}\delta, \omega, V_{t}, P, Q & \begin{array}{l}\text { Synchronous machine (s.m.) torque angle, speed, terminal voltage, } \\ \text { active and reactive power }\end{array} \\ K_{1}, K_{2}, K_{3}, K_{4}, K_{5}, K_{6} & \begin{array}{l}\text { S.m. linear model parameters } \\ \text { s.m. inertia constant, accelerating, electrical and mechanical torque }\end{array} \\ H, T_{a}, T_{e}, T_{m} & \text { Equivalent resistance and reactance of external system } \\ R_{e}, X_{e} & \text { Voltage proportional to d-axis flux linkages, field voltage and } \\ E_{q}^{\prime}, E_{F D}, \tau_{d 0}^{\prime} & \text { open-circuit time constant } \\ K_{E}, S_{E}, \tau_{E} & \text { Self-excited field constant, saturation function and time constant } \\ & \text { of exciter } \\ K_{A}, \tau_{A}, v_{R} & \text { Regulator gain, time constant and output voltage } \\ K_{F}, \tau_{F} & \text { Rate feedback (RF) gain and time constant } \\ K_{R}, \tau_{R} & \text { Transducer/filter gain and time constant } \\ K_{0}, \tau_{0}, V_{s} & \text { Speed gain, reset time-lag constant and voltage output of power } \\ \tau_{1}, \tau_{3}, \tau_{2}, \tau_{4} & \text { system stabilizer (PSS) } \\ S & \text { Lead and lag time constants of PSS } \\ \Delta & \text { Laplace operator } \\ & \text { Incremental (step) change of input }\end{array}$




\section{INTRODUCTION}

Every physical system can be translated into mathematical model. The mathematical procedure of system modelling often leads to comprehensive description of a process in the form of high order differential equations which are difficult to use either for analysis or controller synthesis. It is hence useful, and sometimes necessary, to find the possibility of finding some equation of the same type but of lower order that may be considered to adequately reflect the dominant characteristics of the system under consideration. Some of the reasons for using reduced order models of high order linear systems could be:

- To have a better understanding of the system.

- To reduce computational complexity.

- To reduce hardware complexity.

- To make feasible controller design.

A large number of methods are available in the literature for order reduction of linear continuous systems in time domain as well as in frequency domain [1-7]. The extension of single-input single-output (SISO) methods to reduce multi-input multi-output (MIMO) systems has also been carried out in [8-11]. Each of these methods has both advantages and disadvantages when tried on a particular system. In spite of several methods available, no approach always gives the best results for all systems.

Further, numerous methods of order reduction are also available in the literature [12-17], which are based on the minimization of the integral square error (ISE) criterion. However, a common feature in these methods [12-16] is that the values of the denominator coefficients of the low order system (LOS) are chosen arbitrarily by some stability preserving methods such as dominant pole, Routh approximation methods, etc. and then the numerator coefficients of the LOS are determined by minimization of the ISE. In [17], Howitt and Luss suggested a technique, in which both the numerator and denominator coefficients are considered to be free parameters and are chosen to minimize the ISE in impulse or step responses.

Recently, Genetic algorithm (GA) is becoming popular to solve the optimization problems in different fields of application mainly because of their robustness in finding an optimal solution and ability to provide a near optimal solution close to a global minimum. Unlike strict mathematical methods, the GA does not require the condition that the variables in the optimization problem be continuous and different; it only requires that the problem to be solved can be computed. GA employs search procedures based on the mechanics of natural selection and survival of the fittest. The GAs, which use a multiple point instead of a single point search and work with the coded structure of variables instead of the actual variables, require only the objective function thereby making searching for a global optimum simpler [18-19]. The present attempt is towards evolving a new algorithm for order reduction of linear multivariable systems, which combines the advantages of the dominant pole retention method and the error minimization by GA. Basically; the method starts with fixation of the denominator of the LOS by dominant pole retention method followed by the determination of coefficients of the numerator polynomials of each element of the LOS transfer matrix by minimizing the ISE in between the transient responses of original and LOS using GA. In the following sections, the algorithm is described in detail and the same has been applied to a $10^{\text {th }}$ order two-input two-output linear time invariant model of a practical power system [20]. The performance of the algorithm is tested by comparing the relevant computer simulation results.

\section{DESCRIPTION OF THE ALGORITHM}

Let the transfer function matrix of the high order system (HOS) of order ' $n$ ' having ' $p$ ' inputs and ' $m$ ' outputs be: 


$$
[G(s)]=\frac{1}{D(s)}\left[\begin{array}{ccccc}
a_{11}(s) & a_{12}(s) & a_{13}(s) & \ldots & a_{1 p}(s) \\
a_{21}(s) & a_{22}(s) & a_{23}(s) & \ldots & a_{2 p}(s) \\
\vdots & \vdots & \vdots & \ldots & \vdots \\
a_{m 1}(s) & a_{m 2}(s) & a_{m 3}(s) & \ldots & a_{m p}(s)
\end{array}\right]
$$

or, $\quad[G(s)]=\left[g_{i j}(s)\right], i=1,2, \ldots . ., m ; j=1,2, \ldots \ldots ., p$

is a $m \times p$ transfer matrix.

The general form of $g_{i j}(s)$ of $[G(s)]$ in (1) is taken as:

$$
\begin{aligned}
g_{i j}(s) & =\frac{a_{i j}(s)}{D(s)} \\
& =\frac{a_{o}+a_{1} s+a_{2} s^{2}+\ldots+a_{n-1} s^{n-1}}{b_{o}+b_{1} s+b_{2} s^{2}+\ldots . b_{n-1} s^{n-1}+s^{n}} \\
\text { or, } \quad g_{i j}(s) & =\frac{a_{o}+a_{1} s+a_{2} s^{2}+\ldots+a_{n-1} s^{n-1}}{\left(s+\lambda_{1}\right)\left(s+\lambda_{2}\right) \ldots \ldots\left(s+\lambda_{n}\right)}
\end{aligned}
$$

where, $-\lambda_{1}<-\lambda_{2}<\ldots . .<-\lambda_{n}$ are poles of the HOS.

Let, the transfer function matrix of the LOS of order ' $r$ ' having ' $p$ ' inputs and ' $m$ ' outputs to be synthesized is:

$$
[R(s)]=\frac{1}{\tilde{D}(s)}\left[\begin{array}{ccccc}
b_{11}(s) & b_{12}(s) & b_{13}(s) & \ldots & b_{1 p}(s) \\
b_{21}(s) & b_{22}(s) & b_{23}(s) & \ldots & b_{2 p}(s) \\
\vdots & \vdots & \vdots & \ldots & \vdots \\
b_{m 1}(s) & b_{m 2}(s) & b_{m 3}(s) & \ldots & b_{m p}(s)
\end{array}\right]
$$

or, $\quad[R(s)]=\left[r_{i j}(s)\right], i=1,2, \ldots . ., m ; j=1,2, \ldots . ., p$

is a $m \times p$ transfer matrix.

The general form of $r_{i j}(s)$ of $[R(s)]$ in (4) is taken as:

$$
\begin{aligned}
r_{i j}(s) & =\frac{b_{i j}(s)}{\tilde{D}(s)} \\
& =\frac{\alpha_{0}+\alpha_{1} s+\ldots+\alpha_{r-1} s^{r-1}}{d_{o}+d_{1} s+d_{2} s^{2}+\ldots .+d_{r-1} s^{r-1}+s^{r}}
\end{aligned}
$$

or, $\quad r_{i j}(s)=\frac{\alpha_{0}+\alpha_{1} s+\ldots+\alpha_{r-1} s^{r-1}}{\left(s+\lambda_{1}\right)\left(s+\lambda_{2}\right) \ldots \ldots\left(s+\lambda_{r}\right)}$

where, $-\lambda_{1}<-\lambda_{2}<\ldots . .<-\lambda_{r}$ are the dominant poles of the HOS, then steps are as under.

\subsection{Determination of the denominator coefficients of LOS}

Retention of dominant poles of HOS in LOS [13, 21]:

Depending on the order to be reduced to, the poles nearest to the origin are retained. This implies that the over all behavior of the reduced system will be very similar to the original system, since the contribution of the unretained eigen values to the system response are important only at the beginning of the response, where as the eigen values retained are important throughout the whole of the response, and, infact, determine the type of the response of the system. Therefore, the denominator polynomial in (5) is now known, which is given by: $\tilde{D}(s)=d_{o}+d_{1} s+d_{2} s^{2}+\ldots \ldots+d_{r-1} s^{r-1}+s^{r}$. 


\subsection{Determination of the numerator coefficients of LOS by Genetic Algorithm}

In the present study, GA is employed to minimize the objective function ' $E$ ', which is the integral square error in between the transient responses of original $\left(g_{i j}(s)\right)$ and reduced $\left(r_{i j}(s)\right)$ order models and is given by:

$$
E=\int_{0}^{\infty}\left[g_{i j}(t)-r_{i j}(t)\right]^{2} d t
$$

where, $i=1,2, \ldots, m ; j=1,2, \ldots, p$ and $g_{i j}(t), r_{i j}(t)$ are the unit step responses of original and reduced order models, respectively and the parameters to be determined are the numerator coefficients $\alpha_{i}(i=0,1, \ldots .,(r-1))$ of reduced order models $\left(r_{i j}(s)\right)$ of the LOS $[R(s)]$.

For the purpose of minimization of (7), routines from GAOT toolbox are used. For different problems, it is possible that the same parameters for GA do not give the best solution and so these can be changed according to the situation. In Table I, the typical parameters for the GA optimization routines, used in the present study are given. The description of these operators and their properties can be found in [22]. One more important point that affects the optimal solution more or less is the range for unknowns. For the very first execution of the program, wider solution space can be given and after getting the solution one can shorten the solution space nearer to the values obtained in the previous iteration. The computational flowchart of the GA optimization is shown in Fig. 1.

Table I: Typical parameters used by the Genetic Algorithm.

\begin{tabular}{|l|c|}
\hline \multicolumn{1}{|c|}{ Name } & Value (type) \\
\hline Number of generations & 200 \\
\hline Population size & 50 \\
\hline Type of selection & Normal geometric [0.08] \\
\hline Type of crossover & Arithmetic [2] \\
\hline Type of mutation & Nonuniform [2 200 3] \\
\hline Termination method & Maximum generation \\
\hline
\end{tabular}

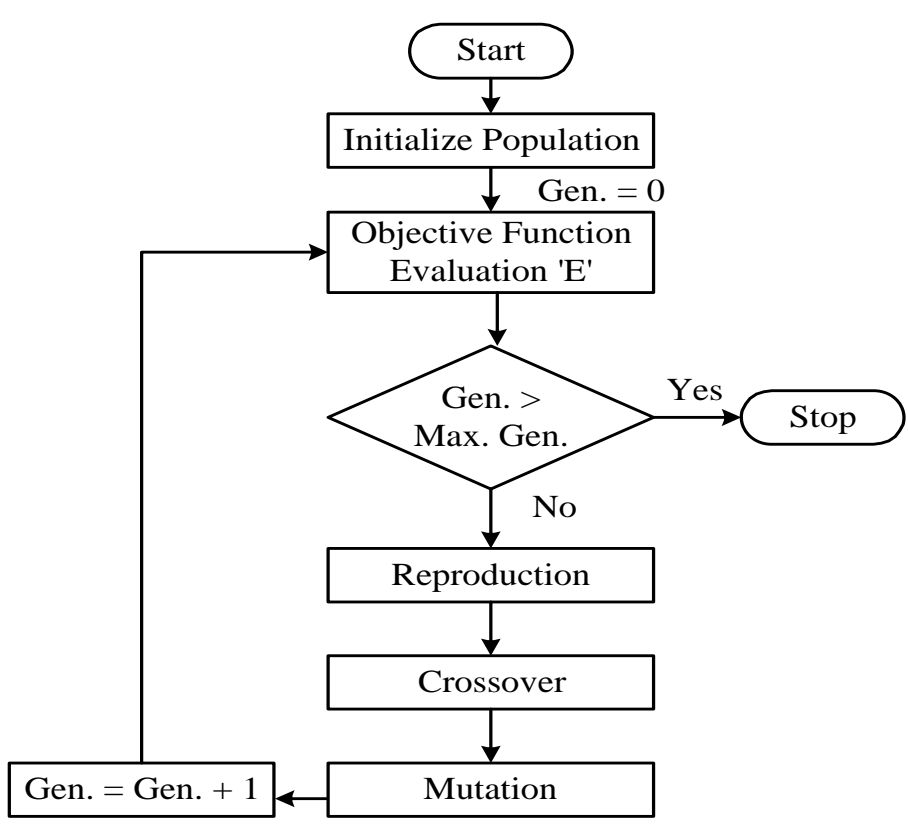

Figure 1: Flowchart of Genetic Algorithm. 


\section{PRACTICAL POWER SYSTEM UNDER STUDY}

The single-machine infinite-bus (SMIB) power system shown in Fig. 2 is considered in this study. The machine is supplying power through a step-up transformer and a high-voltage transmission line to an infinite grid. In the Fig. 2, $X_{T}$ and $X_{L}$ represent the reactance of the transformer and the transmission line respectively; $V_{T}$ and $V_{B}$ are the generator terminal and infinite bus voltage, respectively.

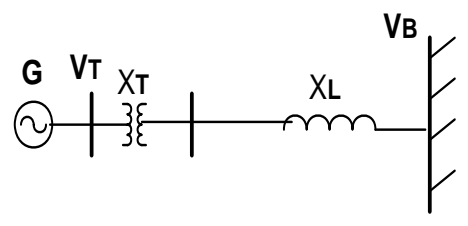

Figure 2: Single-machine infinite-bus (SMIB) power system.

The Phillips-Heffron model of the SMIB power system is shown in Fig. 3. The system consists of a three-phase 160-MVA synchronous machine with automatic excitation control system (i.e. a standard IEEE Type-I exciter with rate feedback (RF) and power system stabilizer (PSS)). This simple synchronous machine model was developed by Heffron and Phillips [23]. The simplicity and overall usefulness of this model continues to find wide use by investigators in power system studies [24]. The numerical values of the parameters, which define the total system as well as its operating point, are given in Appendix A [25].

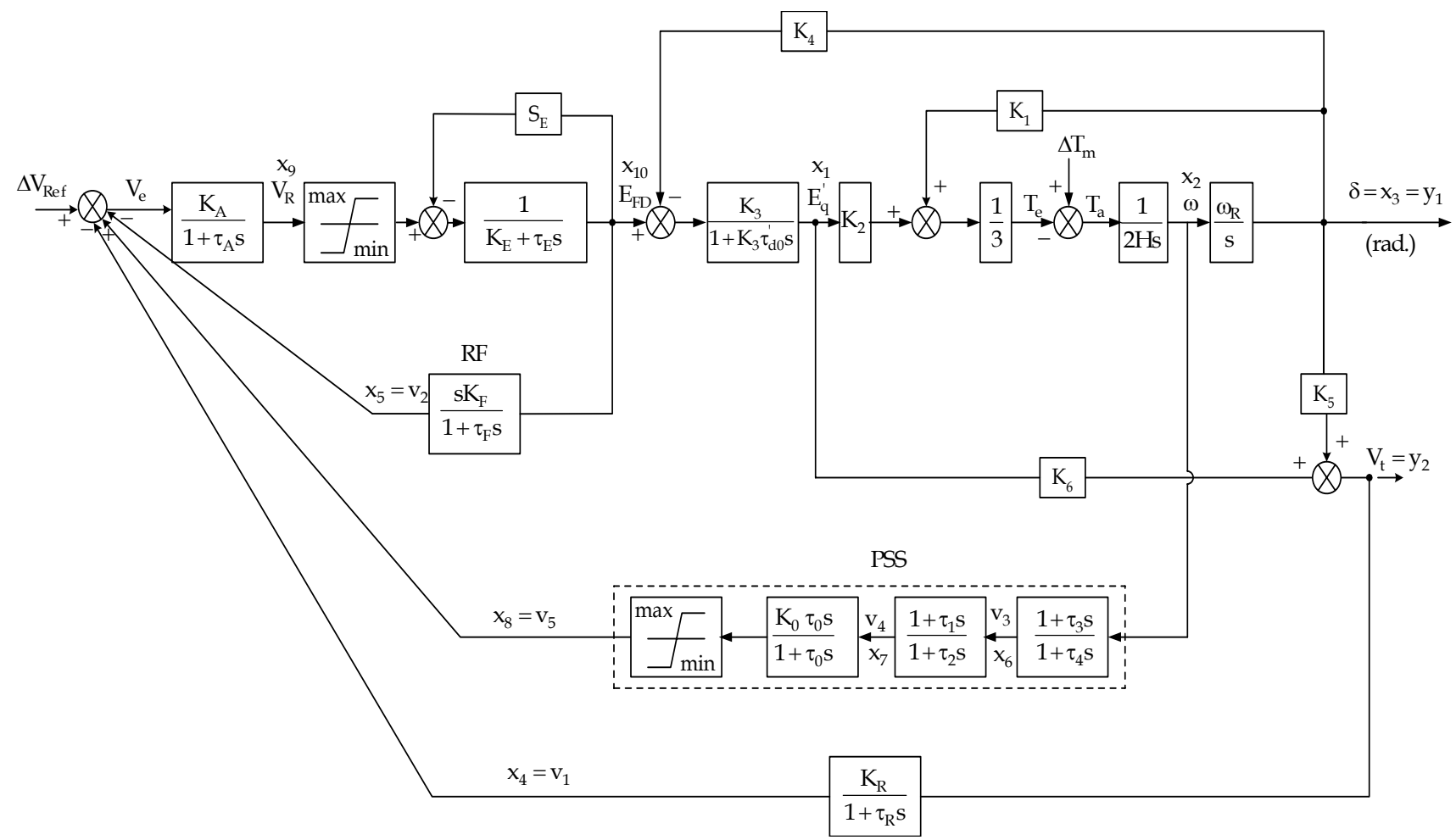

Figure 3: Block diagram representation of Phillips-Heffron model of single-machine infinitebus (SMIB) power system.

Based on the state variables (Fig. 3) and the values of the parameters and the operating point (Appendix A), the system of Fig. 3 (without accounting for the limiters) may be described in state-space form as: 
where,

$$
\left.\begin{array}{l}
\dot{x}=A x+B u \\
y=C x
\end{array}\right\}
$$

$$
x^{T}=\left[\begin{array}{llllllllll}
E_{q}^{\prime} & \omega & \delta & v_{1} & v_{2} & v_{3} & v_{4} & v_{5} & v_{R} & E_{F D}
\end{array}\right] ; u^{T}=\left[\begin{array}{lll}
\Delta V_{\operatorname{Re} f} & \Delta T_{m}
\end{array}\right] ; y^{T}=\left[\begin{array}{ll}
\delta & V_{t}
\end{array}\right]
$$

and the numerical values of the matrices $A, B$ and $C$ are given in the Appendix B.

\section{APPLICATION OF THE PROPOSED ALGORITHM TO THE POWER SYSTEM AND SIMULATION RESULTS}

The transfer function matrix (based on the numerical values of the matrices $A, B$ and $C$ ) of the $10^{\text {th }}$ order two-input two-output linear time invariant model of practical power system under study is given by :

$$
[G(s)]=\frac{1}{D(s)}\left[\begin{array}{ll}
a_{11}(s) & a_{12}(s) \\
a_{21}(s) & a_{22}(s)
\end{array}\right]
$$

where, the common denominator $D(s)$ is given by :

$$
\begin{aligned}
D(s)=s^{10}+64.21 s^{9} & +1596 s^{8}+1.947 \times 10^{4} s^{7}+1.268 \times 10^{5} s^{6}+5.036 \times 10^{5} s^{5} \\
& +1.569 \times 10^{6} s^{4}+3.24 \times 10^{6} s^{3}+4.061 \times 10^{6} s^{2}+2.905 \times 10^{6} s+2.531 \times 10^{5}
\end{aligned}
$$

and

$$
\begin{array}{r}
a_{11}(s)=-2298 s^{5}-9.845 \times 10^{4} s^{4}-1.376 \times 10^{6} s^{3}-6.838 \times 10^{6} s^{2}-6.1 \times 10^{6} s-5.43 \times 10^{5} \\
a_{12}(s)=29.09 s^{8}+1868 s^{7}+4.61 \times 10^{4} s^{6}+5.459 \times 10^{5} s^{5}+3.185 \times 10^{6} s^{4}+8.702 \times 10^{6} s^{3} \\
+1.206 \times 10^{7} s^{2}+7.606 \times 10^{6} s+6.483 \times 10^{5} \\
a_{21}(s)=85.23 s^{7}+3651 s^{6}+5.208 \times 10^{4} s^{5}+2.98 \times 10^{5} s^{4}+8.471 \times 10^{5} s^{3}+3.105 \times 10^{6} s^{2} \\
+2.752 \times 10^{6} s+2.45 \times 10^{5} \\
a_{22}(s)=-1.26 s^{8}-85.18 s^{7}-2089 s^{6}-2.568 \times 10^{4} s^{5}-1.909 \times 10^{5} s^{4}-7.123 \times 10^{5} s^{3} \\
-1.084 \times 10^{6} s^{2}-2.972 \times 10^{5} s-1.942 \times 10^{4}
\end{array}
$$

The poles of the above system $[G(s)]$ are at:

$$
\begin{aligned}
& \lambda_{1}=-0.1001, \lambda_{2,3}=-0.2392 \pm j 3.2348, \lambda_{4,5}=-0.8977 \pm j 1.3552, \\
& \lambda_{6}=-2.1375, \lambda_{7}=-9.6454, \lambda_{8}=-11.9632, \lambda_{9,10}=-19.0451 \pm j 2.4859 .
\end{aligned}
$$

The proposed algorithm is applied to the above multivariable system and the reduced order models $\left(r_{i j}(s)\right)$ of the LOS $[R(s)]$ are obtained. The general form of $3^{\text {rd }}$ order reduced transfer function matrix is taken as:

$$
[R(s)]=\frac{1}{\tilde{D}(s)}\left[\begin{array}{ll}
b_{11}(s) & b_{12}(s) \\
b_{21}(s) & b_{22}(s)
\end{array}\right]
$$

where, $\tilde{D}(s)=s^{3}+0.5785 s^{2}+10.5690 s+1.0532$.

and

$$
\begin{aligned}
& b_{11}(s)=7.4 s^{2}-24 s-2.3, \quad b_{12}(s)=0.625056 s^{2}+28.901348 s+2.674504, \\
& b_{21}(s)=-0.616110 s^{2}+7.954828 s+1.032787, \quad b_{22}(s)=-1.5073 s^{2}-2.9999 s-0.0808
\end{aligned}
$$

The poles of the $\operatorname{LOS}[R(s)]$ are at $\lambda_{1}=-0.1001, \lambda_{2,3}=-0.2392 \pm j 3.2348$. 
The adequacy of the $3^{\text {rd }}$ order reduced models obtained above is tested by comparing the time responses of the outputs (i.e. $\delta$ and $V_{t}$ ) of the original $10^{\text {th }}$ order system (9) and those of the $3^{\text {rd }}$ order reduced system (10), subject to the same input step change. The time responses shown in Fig. 4 (a)-(f) are also compared with the $3^{\text {rd }}$ order reduced models obtained by Papadopoulos and Boglou [20] and are computed for three distinct input step changes:

- with $\Delta V_{\operatorname{Re} f}(s)=0.05$ p.u. and $\Delta T_{m}(s)=0$;

- with $\Delta V_{\operatorname{Re} f}(s)=0$ and $\Delta T_{m}(s)=0.05$ p.u. ; and

- with $\Delta V_{\operatorname{Re} f}(s)=0.05$ p.u. and $\Delta T_{m}(s)=0.05$ p.u.

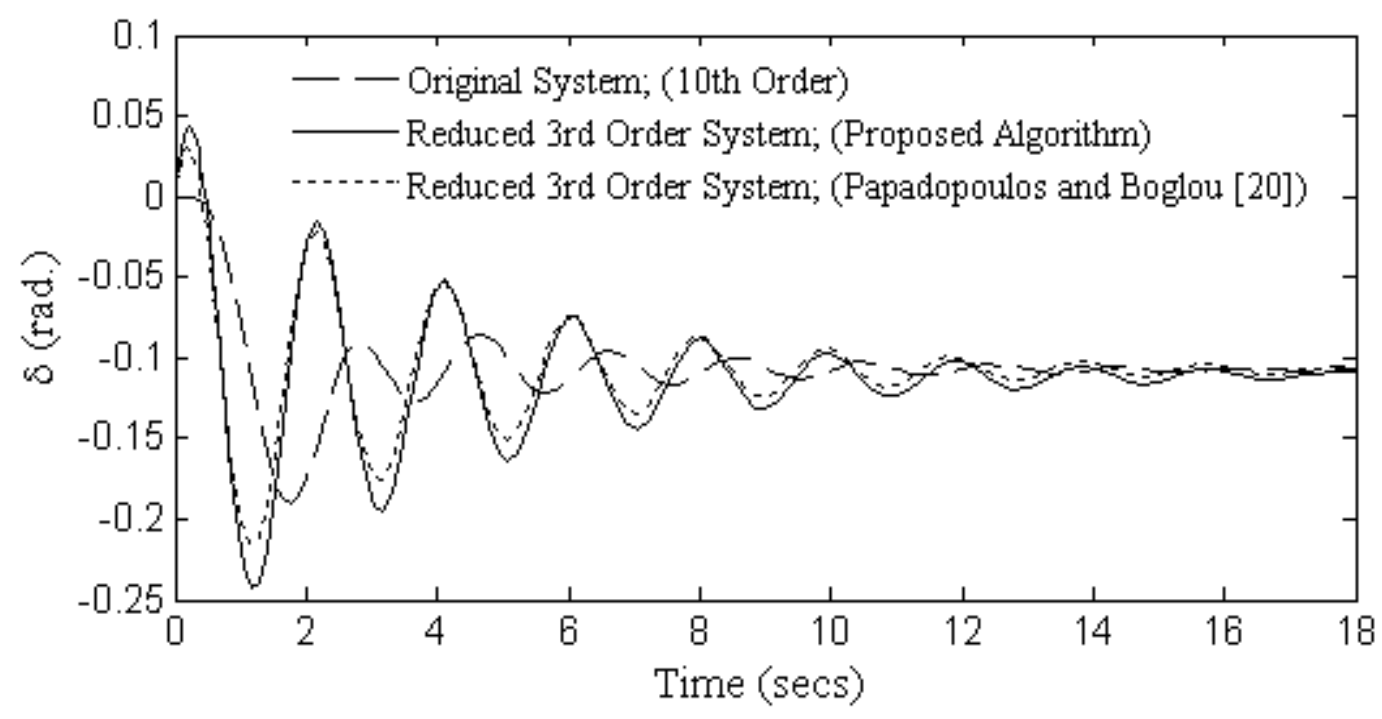

Figure 4 (a): $\Delta V_{\operatorname{Re} f}=0.05$ and $\Delta T_{m}=0$ p.u.

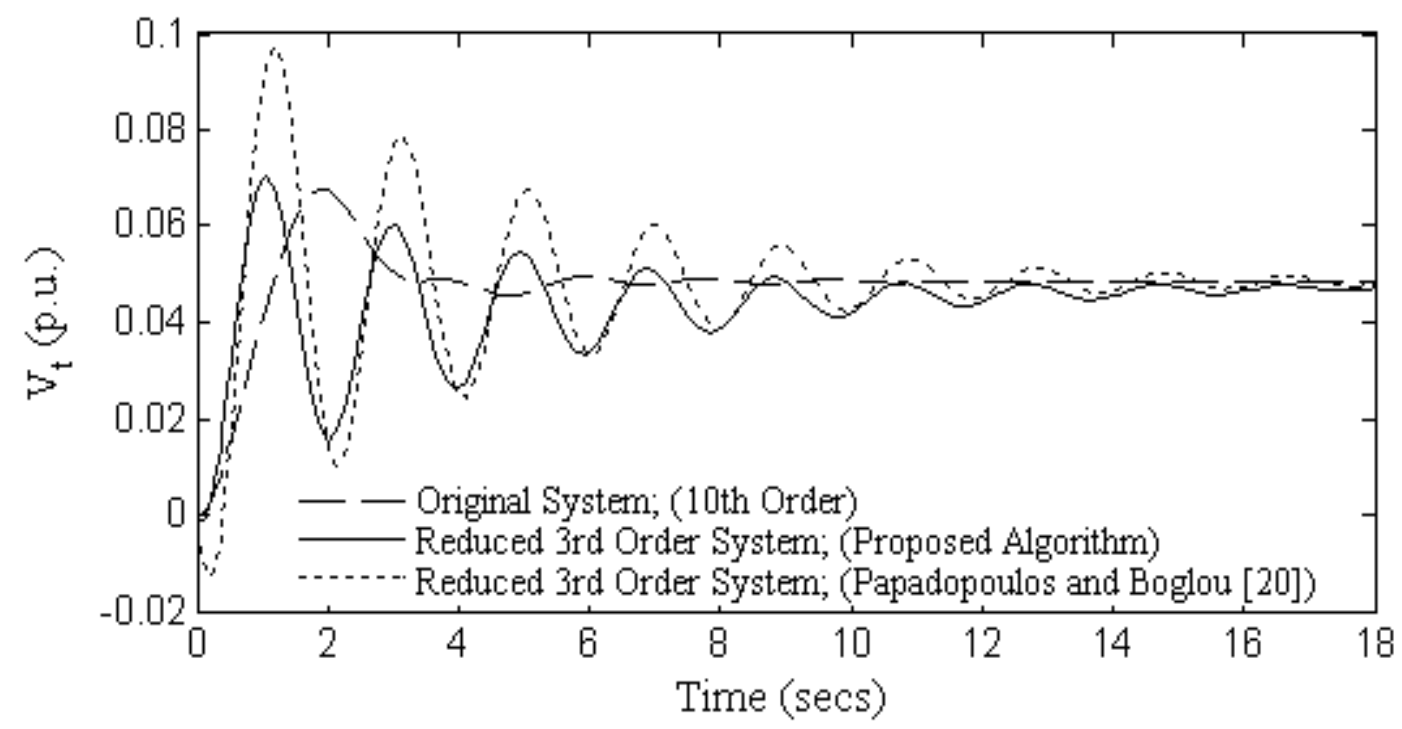

Figure 4 (b): $\Delta V_{\operatorname{Re} f}=0.05$ and $\Delta T_{m}=0$ p.u. 


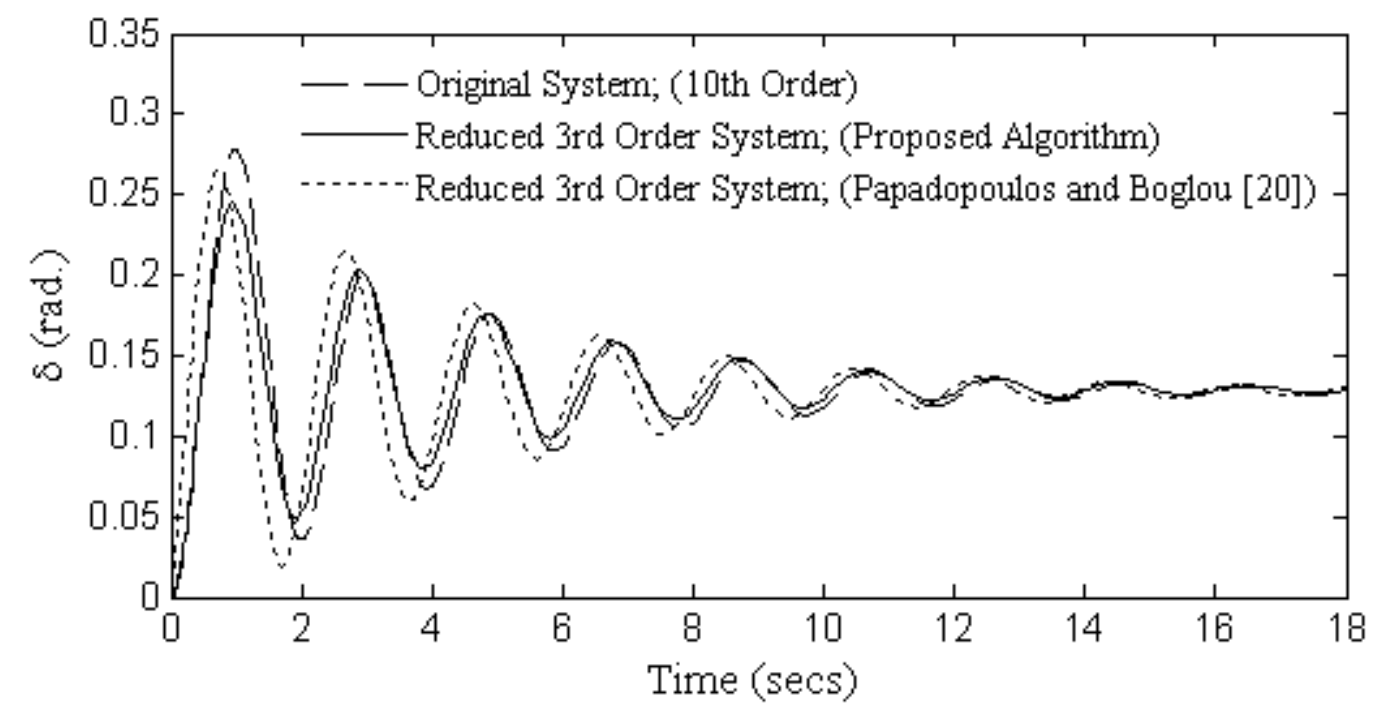

Figure 4 (c): $\Delta V_{\operatorname{Re} f}=0$ and $\Delta T_{m}=0.05$ p.u.

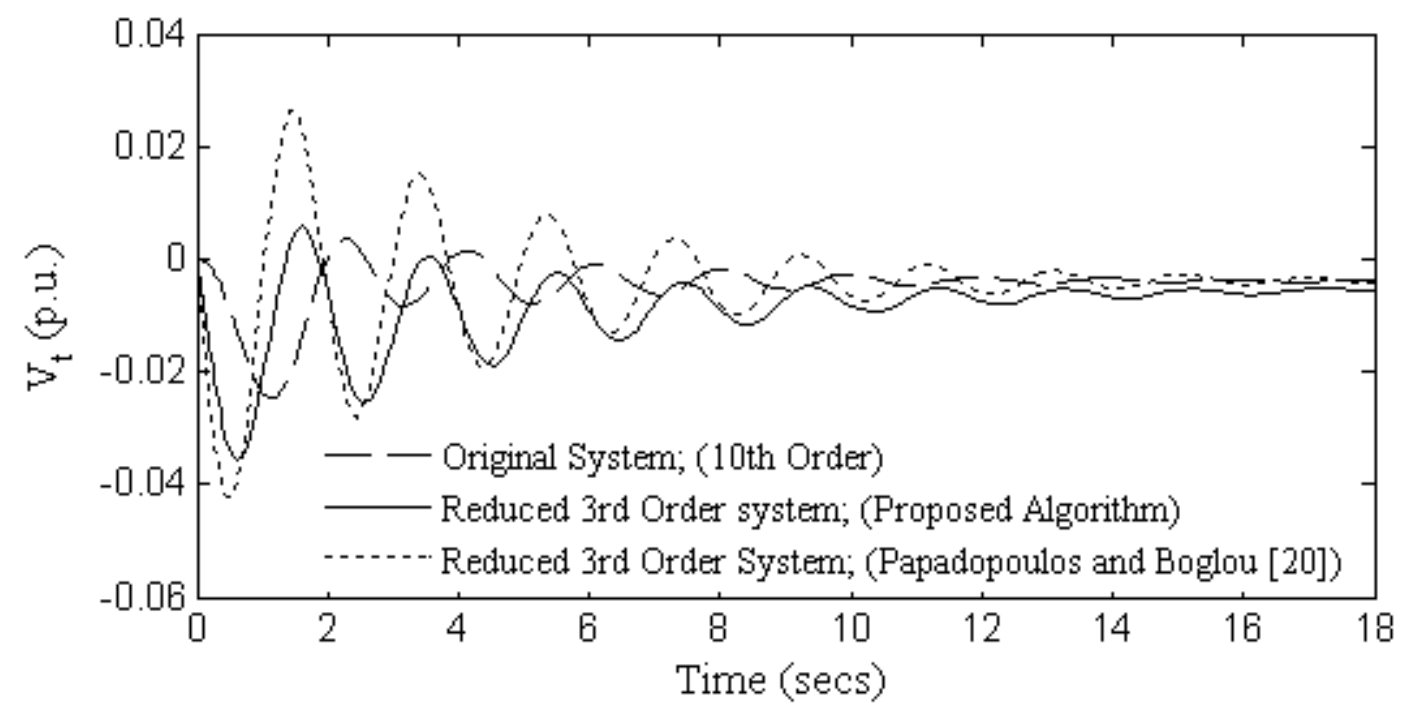

Figure 4 (d): $\Delta V_{\operatorname{Re} f}=0$ and $\Delta T_{m}=0.05$ p.u.

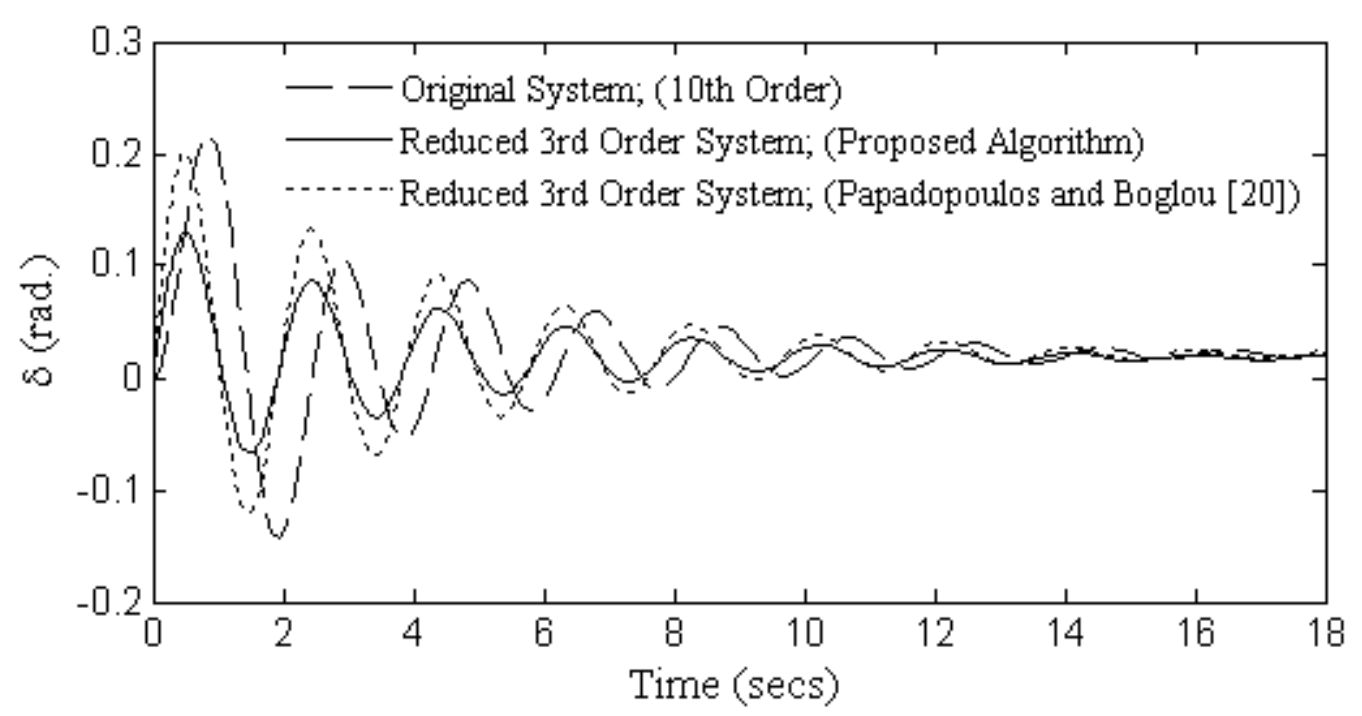

Figure 4 (e): $\Delta V_{\operatorname{Re} f}=0.05$ and $\Delta T_{m}=0.05$ p.u. 


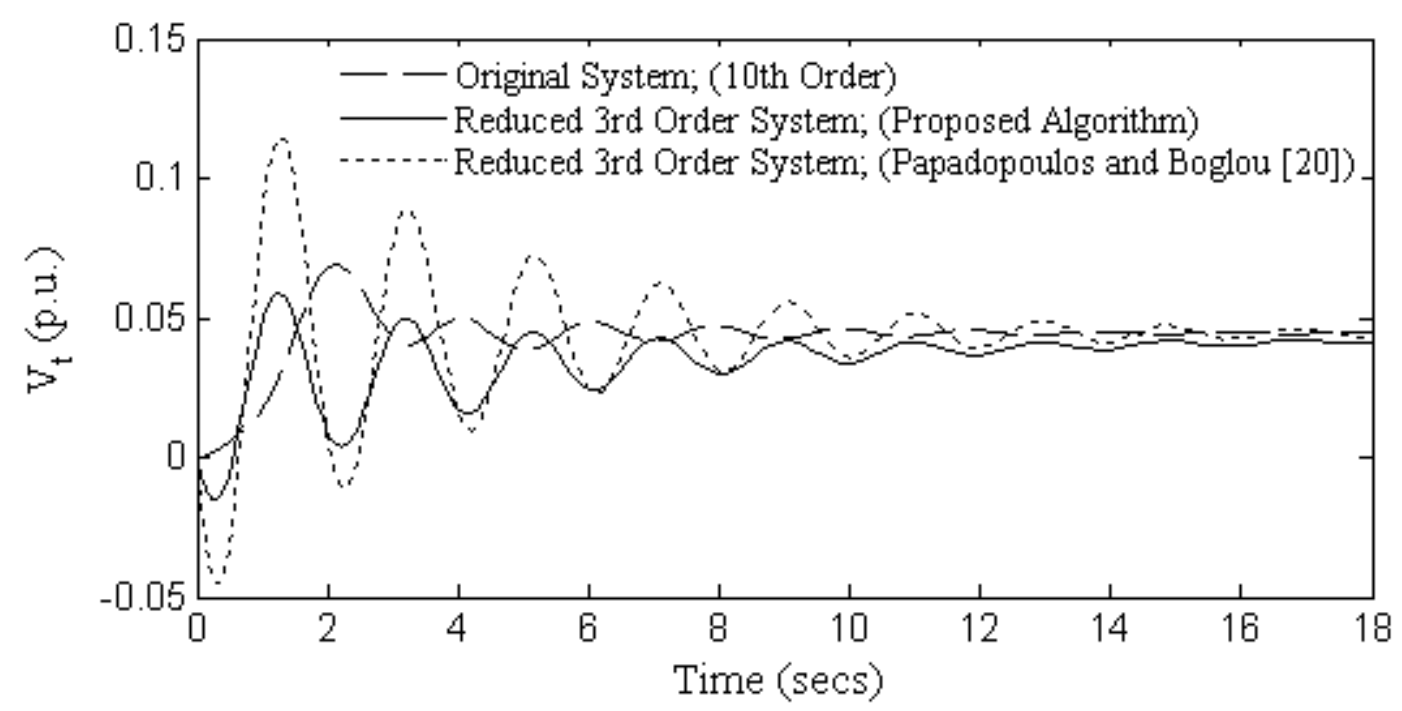

Figure 4 (f): $\Delta V_{\operatorname{Re} f}=0.05$ and $\Delta T_{m}=0.05$ p.u.

From the simulation results shown in Fig. 4 (a)-(f), it is clear that the $3^{\text {rd }}$ order reduced system (10) obtained by the proposed algorithm is adequate since the time responses of its outputs coincide quite well with those of the original $10^{\text {th }}$ order system for the same input step change.

\section{COMPARISON OF THE METHODS}

A comparison of the proposed algorithm with some well known existing order reduction techniques (for $3^{\text {rd }}$ order reduced models), is also shown as given in Table II. An error index R.I.S.E., known as relative integral square error [26] in between the transient parts of original $\left(g_{i j}(s)\right)$ and reduced $\left(r_{i j}(s)\right)$ order models is calculated to measure the goodness of the LOS (i.e. the smaller the R.I.S.E., the closer is $r_{i j}(s)$ to $\left.g_{i j}(s)\right)$. This R.I.S.E. is calculated for each element $\left(r_{i j}(s)\right)$ of the transfer function matrix of the $\operatorname{LOS}[R(s)]$, and it is given by:

$$
J=\int_{0}^{\infty}\left[g_{i j}(t)-r_{i j}(t)\right]^{2} d t / \int_{0}^{\infty}\left[g_{i j}(t)-g_{i j}(\infty)\right]^{2} d t
$$

where, $i=1,2 ; j=1,2$.

and $g_{i j}(t), r_{i j}(t)$ are the unit step responses of original and reduced order models, respectively.

The proposed algorithm has been compared with these techniques [27-30] due to the following reasons :

- These techniques provide explicit error bounds for the reduction-error.

- These techniques are generally considered to be the standard to measure goodness of the linear model reduction.

- These techniques are easy to implement as standard routines are freely available on the web [31] or are built into Matlab toolboxes.

The proposed algorithm has also been compared with Papadopoulos and Boglou [20].

It can be seen in Table II that the proposed algorithm gives low value of $J$ for all values of $r_{i j}(i=1,2 ; j=1,2)$ in comparison to the other existing techniques. 
Table II: Comparison of reduced order models.

\begin{tabular}{|l|c|c|c|c|}
\hline \multicolumn{5}{|c|}{ R.I.S.E. $J$ for $r_{i j}(i=1,2 ; j=1,2)$} \\
\hline Method of Order Reduction & $r_{11}$ & $r_{12}$ & $r_{21}$ & $r_{22}$ \\
\hline Proposed Algorithm $\left[g_{i j}(t)-r_{i j}(t)\right]^{2} d t / \int_{0}^{\infty}\left[g_{i j}(t)-g_{i j}(\infty)\right]^{2} d t$ & 0.978182 \\
\hline Papadopoulos and Boglou [20] & 0.995330 & 0.069978 & 1.919841 & 1.001437 \\
\hline Moore [27] & 1.000215 & 0.683081 & 2.854987 & 0.872135 \\
\hline Safonov and Chiang [28] & 0.939453 & 0.568192 & 3.953609 & 0.034152 \\
\hline Safonov and Chiang [29] & 1.324429 & 205.8792 & 79.98665 & 0.0372409 \\
\hline Safonov, Chiang and Limebeer [30] & 0.943603 & 0.047862 & 17.445711 & 1.057315 \\
\hline
\end{tabular}

\section{CONCLUSIONS}

A new algorithm for the order reduction of linear multivariable systems has been presented in which the dominant poles are retained according to the order to be reduced to, while the Genetic algorithm has been used to determine the zeros by minimizing the integral square error between the transient responses of original and reduced order models. Each element of the transfer function matrix of the original system is considered separately. The algorithm is simple, rugged and computer oriented. The algorithm has been implemented in Matlab 7.0 on a Pentium-IV processor and the computation time is negligible being less than 1 minute. The algorithm has been applied successfully to a $10^{\text {th }}$ order two-input two-output linear time invariant model of a practical power system.

The adequacy of the low order models obtained by the proposed algorithm has been judged by comparing the time responses of their outputs to the corresponding ones of the original system model. A comparison of the proposed algorithm with some well known existing order reduction techniques is also shown as given in Table II, from which it is clear that the proposed algorithm is comparable in quality with the other existing techniques.

In the present work, the GA combined with the dominant pole retention method has been applied for the order reduction of a power system model. The results coming out are quite encouraging but, more work is being carried out by us in this area and further investigations are required before reporting the same. A comparative study of the proposed algorithm with the other optimization techniques available in the literature such as Particle swarm, Ant colony, etc. is being carried out, which is also one of our future works.

\section{REFERENCES}

[1] Genesio, R.; Milanese, M. (1976). A note on the derivation and use of reduced order models, IEEE Transactions on Automatic Control, Vol. AC-21, No. 1, 118-122

[2] Mahmoud, M. S.; Singh, M. G. (1981). Large Scale Systems Modelling, 1st Ed., International Series on Systems and Control, Vol. 3, Pergamon Press

[3] Jamshidi, M. (1983). Large Scale Systems Modelling and Control Series, Vol. 9, North Holland, Amsterdam, Oxford

[4] Nagar, S. K.; Singh, S. K. (2004). An algorithmic approach for system decomposition and balanced realized model reduction, Journal of the Franklin Institute, Vol. 341, 615-630

[5] Singh, V.; Chandra, D.; Kar, H. (2004). Improved Routh Pade approximants: A computer aided approach, IEEE Transactions on Automatic Control, Vol. 49, No. 2, 292-296 
[6] Mukherjee, S.; Satakshi; Mittal, R. C. (2005). Model order reduction using response-matching technique, Journal of the the Franklin Institute, Vol. 342, 503-519

[7] Salimbahrami, B.; Lohmann, B. (2006). Order reduction of large scale second-order systems using Krylov subspace methods, Linear Algebra and its Applications, Vol. 415, pp. 385-405

[8] Mukherjee, S.; Mishra, R. N. (1988). Reduced order modelling of linear multivariable systems using an error minimization technique, Journal of the Franklin Institute, Vol. 325, No. 2, 235 245

[9] Lamba, S. S.; Gorez, R.; Bandyopadhyay, B. (1988). New reduction technique by step error minimization for multivariable systems, International Journal of Systems Science, Vol. 19, No. 6, 999-1009

[10] Prasad, R.; Pal, J. (1991). Use of continued fraction expansion for stable reduction of linear multivariable systems, Journal of Institution of Engineers, India, IE(I) Journal - EL, Vol. 72, 43-47

[11] Prasad, R.; Mittal, A. K.; Sharma, S. P. (2005). A mixed method for the reduction of multivariable systems, Journal of Institution of Engineers, India, IE(I) Journal - EL, Vol. 85, 177-181

[12] Hwang, C.; Wang, K. Y. (1984). Optimal Routh approximations for continuous-time systems, International Journal of Systems Science, Vol. 15, No. 3, 249-259

[13] Mukherjee, S.; Mishra, R. N. (1987). Order reduction of linear systems using an error minimization technique, Journal of the Franklin Institute, Vol. 323, No. 1, 23-32

[14] Puri, N. N.; Lan, D. P. (1988). Stable model reduction by impulse response error minimization using Mihailov criterion and Pade's approximation, Transactions ASME, Journal of Dynamic System Measurement and Control, Vol. 110, pp. 389-394

[15] Vilbe, P.; Calvez L. C. (1990). On order reduction of linear systems using an error minimization technique, Journal of the Franklin Institute, Vol. 327, pp. 513-514

[16] Mittal, A. K.; Prasad, R.; Sharma, S. P. (2004). Reduction of linear dynamic systems using an error minimization technique, Journal of Institution of Engineers, India, IE(I) Journal - EL, Vol. 84, 201-206

[17] Howitt, G. D.; Luus, R. (1990). Model reduction by minimization of integral square error performance indices, Journal of the Franklin Institute, Vol. 327, pp. 343-357

[18] Deb, K. (1998). Optimization for Engineering Design: Algorithms and Examples, Prentice Hall, India

[19] Goldberg, D. E. (2003). Genetic Algorithms in Search, Optimization \& Machine Learning, Pearson Education, India

[20] Papadopoulos, D. P.; Boglou, A. K. (1990). Reduced-order modelling of linear MIMO systems with the Pade approximation method, International Journal of Systems Science, Vol. 21, No. 4, 693-710

[21] Davison, E. J. (1966). A method for simplifying linear dynamic systems, IEEE Transactions on Automatic Control, Vol. AC-11, 93-101

[22] Houck, C.; Joines, J.; Kay, M. (1995). A Genetic Algorithm for Function Optimization: A Matlab Implementation, NCSU-IE TR 95-05,

[23] From http://www.ise.ncsu.edu/mirage/GAToolBox/gaot, accessed on 20-03-2005

[24] Heffron, W. G.; Phillips, R. A. (1952). Effect of modern amplidyne voltage regulators on underexcited operation of large turbine generators, AIEE Transactions, Vol. PAS-71, pp. 692697

[25] Papadopoulos, D. P. (1986). Order reduction of linear system models with a time-frequency domain method, Journal of the Franklin Institute, Vol. 322, No. 4, pp. 209-220

[26] Papadopoulos, D. P. (1982). Tests and simulations in connection with large generator dynamics, Proceedings of the ICEM'82 Conference, Budapest, Part 3, 881-884

[27] Lucas, T. N. (1987). Further discussion on impulse energy approximation, IEEE Transactions on Automatic Control, Vol. AC-32, No. 2, 189-190

[28] Moore, B. C. (1981). Principal component analysis in linear systems: controllability, observability and model reduction, IEEE Transactions on Automat. Control, Vol. AC-26, No. 1, $17-32$ 
[29] Safonov, M. G.; Chiang, R. Y. (1988). Model reduction for robust control: A Schur relative error method, International Journal of Adaptive Control and Signal Processing, Vol. 2, 259-272

[30] Safonov, M. G.; Chiang, R. Y. (1989). A Schur method for balanced-truncation model reduction, IEEE Transactions on Automatic Control, Vol. 34, No.7, 729-733

[31] Safonov, M. G.; Chiang, R. Y.; Limebeer D. J. N. (1990). Optimal Hankel model reduction for nonminimal systems, IEEE Transactions on Automatic Control, Vol. 35, No. 4, 496-502

[32] Varga, A. (2001). Model reduction software in the SLICOT library, in Applied and computational control, signals and circuits, Ed. B. Datta, vol. 629, Kluwer Academic Publishers, Boston. From http://www.robotic.dlr.de/ varga/, accessed on 23-11-2004

\section{APPENDICES}

Appendix A. Numerical values of system parameters and operating point.

Synchronous machine : 3-phase, 160 MVA, pf $=0.894, x_{d}=1.7, x_{q}=1.6, x_{d}^{\prime}=0.245$ p.u., $\tau_{d o}^{\prime}=5.9, \mathrm{H}=5.4 \mathrm{~s}, \omega_{R}=314 \mathrm{rad} \mathrm{s}^{-1}$.

Type-I exciter: $K_{A}=50, K_{E}=-0.17, S_{E}=0.95, K_{F}=0.04, K_{R}=1, K_{0}=1, \tau_{A}=0.05, \tau_{E}=0.95$, $\tau_{F}=1.0, \tau_{R}=0.05, \tau_{0}=10.0, \tau_{1}=\tau_{3}=0.440, \tau_{2}=\tau_{4}=0.092 \mathrm{~s}$.

External System : $R_{e}=0.02, X_{e}=0.40$ p.u. (on 160 MVA base).

Operating Point : $P_{o}=1.0, Q_{0}=0.5, E_{F D_{0}}=2.5128, E_{q 0}=0.9986, v_{t_{0}}=1.0, T_{m_{0}}=1.0$ p.u., $\delta_{0}=1.1966 \mathrm{rad}, K_{1}=1.1330, K_{2}=1.3295, K_{3}=0.3072, K_{4}=1.8235, K_{5}=-0.0433, K_{6}=0.4777$.

Appendix B. Numerical values of matrices $A, B$ and $C$ of the original $10^{\text {th }}$ order system.

$A=\left[\begin{array}{llllllllll}-0.5517 & 0 & -0.3091 & 0 & 0 & 0 & 0 & 0 & 0 & 0.1695 \\ -0.0410 & 0 & -0.0350 & 0 & 0 & 0 & 0 & 0 & 0 & 0 \\ 0 & 314.1593 & 0 & 0 & 0 & 0 & 0 & 0 & 0 & 0 \\ 9.5540 & 0 & -0.8660 & -20 & 0 & 0 & 0 & 0 & 0 & 0 \\ 0 & 0 & 0 & 0 & -1 & 0 & 0 & 0 & 0.0421 & -0.0328 \\ -0.1962 & 10.8696 & -0.1672 & 0 & 0 & -10.8696 & 0 & 0 & 0 & 0 \\ -0.9386 & 51.9849 & -0.7999 & 0 & 0 & -41.1153 & -10.8696 & 0 & 0 & 0 \\ -0.9386 & 51.9849 & -0.7999 & 0 & 0 & -41.1153 & -10.8696 & -0.1 & 0 & 0 \\ 0 & 0 & 0 & -1000 & -1000 & 0 & 0 & 1000 & -20 & 0 \\ 0 & 0 & 0 & 0 & 0 & 0 & 0 & 0 & 1.0526 & -0.8211\end{array}\right]$

$B=\left[\begin{array}{ll}0 & 0 \\ 0 & 0.0926 \\ 0 & 0 \\ 0 & 0 \\ 0 & 0 \\ 0 & 0.4428 \\ 0 & 2.1179 \\ 0 & 2.1179 \\ 1000 & 0 \\ 0 & 0\end{array}\right] ; \quad C=\left[\begin{array}{llllllllll}0 & 0 & 1 & 0 & 0 & 0 & 0 & 0 & 0 & 0 \\ 0.4777 & 0 & -0.0433 & 0 & 0 & 0 & 0 & 0 & 0 & 0\end{array}\right]$ 